\title{
Post-operative continous epidural infusion in geriatric patients with cardiopulmonary co- morbidities: comparison between ropivacaine and levobupivacaine
}

\begin{abstract}
Background: With the increasing life expectancy, the attention of healthcare professionals is being drawn towards issues that affect the elderly population. Post-operative pain management among elderly patients with co-morbidities is a challenging and daunting task for anesthesiologists. In the recent years, regional anesthesia has emerged as one of the preferred and convenient mode of post-operative analgesic management in elderly patients owing to its advantage of not interfering with the metabolic functions, better tolerability and decrease in reflex activity and sensitivity to pain.
\end{abstract}

Methods: In this prospective randomized study, 56 elderly patients with cardiopulmonary comorbidities under ASA-2 and ASA-3 scheduled for elective orthopedic lower limb surgery were enrolled in the study and were randomly allocated to two groups for post operative epidural analgesia consisting 28 patients each as under: Group I: Epidural analgesia Ropivacaine $0.2 \% @ 6 \mathrm{ml} / \mathrm{hr}$, Group II: Epidural analgesia Levobupivicaine $0.2 \%$ (a) $6 \mathrm{ml} / \mathrm{hr}$.

Statistical evaluation: Hemodynamic profiles, Onset of analgesia, extent of sensory and motor block were recorded. To compare the change in a parameter at two different time intervals paired " $\mathrm{t}$ " test was used. Block characteristics were analyzed using Mann Whitney U test.

Results: The findings in present study showed a comparable profile of ropivacaine and levobupivacaine on most of the counts. From clinical point of view, both provided excellent analgesic effect, a good hemodynamic stability and complication free infusion. As compared to levobupivacaine, ropivacaine had an early motor block recovery, early achievement of VAS score 0 , ability to achieve higher levels of block and finally prolongation of analgesic effect even after the infusion was stopped.

Conclusions: As compared to levobupivacaine, ropivacaine seems to be a better option for continuous epidural infusion owing to early motor block recovery, and prolonged analgesia after the infusion was stopped. The findings in present study are specific in view of the specific population they addressed, there is no doubt that cognition issues related with objective scoring of VAS, loss of tactile sensation owing to probable systemic illnesses and growing age might have a confounding effect on the results.
Volume 3 Issue 3 - 2015

Furkan Akhtar Ansari,' Shilpi Misra²

'Junior Resident, Department of Anaesthesiology, India

${ }^{2}$ Associate Professor, Department of Anaesthesiology, India

Correspondence: Shilpi Misra, Associate Professor, Department of Anaesthesiology, Integral Institute of Medical Sciences and Research Lucknow, 39 Chandganj Garden, Aliganj, Lucknow, Uttar Pradesh, India, Tel +9l 9839932239, Email drshilpil980@gmail.com

Received: November 01, 2015 | Published: November 13, 2015

\section{Introduction}

With increasing life expectancy, improved healthcare and increasing prosperity, the proportion of elderly population is increasing throughout the world. ${ }^{1}$ Altered redistribution kinetics as well as compromised drug clearance capacity render geriatric patients particularly vulnerable to drug-induced complications. ${ }^{2}$ Regional anesthesia is preferred for the older patients because this form of anesthesia causes the least interference with the metabolic functions. Geriatric patients, as a rule, tolerate regional anesthesia rather well because of their decrease in autonomic and somatic reflex activity and sensitivity to pain. Bupivacaine, the widely used local anesthetic in regional anesthesia is available in a commercial preparation as a racemic mixture (50:50) of its two enantiomers, levobupivacaine, $\mathrm{S}(-)$ isomer and dextrobupivacaine, $\mathrm{R}(+)$ isomer. Severe central nervous system (CNS) and cardiovascular adverse reactions reported in the literature after inadvertent intravascular injection have been linked to the $\mathrm{R}(+)$ isomer of bupivacaine. The pure $\mathrm{S}(-)$ enantiomers of bupivacaine, i.e., ropivacaine and levobupivacaine were thus introduced into the clinical anesthesia practice. Considering the relatively better cardio toxicity profile, both levobupivacaine as well as ropivacaine are suitable for use as continuous epidural post-operative analgesics in elderly patients with cardiopulmonary comorbidities. However, given the scarcity of clinical comparative data on the issue, it is essential that a comparative study using both the drugs should be carried out. The present study is undertaken to evaluate the efficacy of ropivacaine $0.2 \%$ versus levobupivacaine $0.2 \%$ infusion at the rate of $6 \mathrm{ml} / \mathrm{hr}$, for continuous post-operative epidural analgesia in elderly patients with cardiopulmonary co-morbidities.

\section{Material and methods}

This was a prospective randomized controlled trial approved by institutional ethical committee. An individual informed consent was taken from all the patients enrolled in the study. The following inclusion and exclusion criteria was used: 


\section{A. Inclusion Criteria}

a. Elderly patients aged $>60$ years with cardiopulmonary comorbidities

b. Patients scheduled for elective orthopedic lower limb surgery under epidural anesthesia with continuous epidural infusion for post-operative analgesia.

\section{B. Exclusion Criteria}

a. Patient refusal.

b. Patients with renal failure, hepatic dysfunction, Neuromusculardisorder, Morbidobesity, Bleeding disorders, Infections.

c. History of allergy or sensitivity to any of the studied local anesthetics for previous surgeries.

56 elderly patients with cardiopulmonary comorbidities under ASA-2 and ASA-3 scheduled for elective orthopaedic lower limb surgery under epidural anaesthesia with continuous post-operative epidural analgesia were enrolled in the study and were randomly allocated to two groups for post operative epidural analgesia consisting 28 patients each as under: Group I: Epidural analgesia Ropivacaine $0.2 \% @ 6 \mathrm{ml} / \mathrm{hr}$, Group II: Epidural analgesia Levobupivicaine 0.2\% (a) $6 \mathrm{ml} / \mathrm{hr}$.

In Group I, Out of 19 cases of ASA Grade II, 15 cases were hypertensive, 4 were COPD Grade I. Out of 9 cases of ASA Grade III, 6 cases were of CAD and rest 3 cases were COPD Grade II \& III. In Group II, Out of 19 cases of ASA Grade II, 14 cases were hypertensive, 5 were COPD Grade I. Out of 9 cases of ASA Grade III, 7 cases were of CAD and rest 2 cases were COPD Grade II \& III. The Home Pump Eclipse Ambulatory Infusion System (C300060) of volume $300 \mathrm{ml}$ with flow rate of $6 \mathrm{ml} /$ hour was used in the study. They have elastomeric membrane that assures outstanding performance and reliability.

Patients were asked to nil per oral for solid food 6 hours before surgery and nil per oral for clear liquid for 2 hours before surgery. Patients were premedicated with Tablet Diazepam 5mg and Tablet Ranitidine $150 \mathrm{mg}$ in the night before surgery. After wheeling the patient into operation theatre, a good IV access was secured and patients were connected to all non-invasive monitors for baseline parameters including pulse rate, non invasive blood pressure (NIBP) and Oxygen saturation (SpO2), ECG and Respiratory Rate (RR).

After proper positioning and under strict aseptic precautions local infiltration of Lignocaine $2 \% 2 \mathrm{ml}$ at the puncture site, 18 gauge Tuohy's needle was inserted into the L2-3 interspinal epidural space. Epidural space was confirmed by the loss of resistance method by $10 \mathrm{ml} \mathrm{L.O.R.} \mathrm{syringe.} \mathrm{After} 5$ minutes of institution of test dose ( $3 \mathrm{ml}$ injection lignocaine with adrenaline $2 \%$ ) epidural catheter is inserted and fixed. The scheduled surgery was performed under epidural anesthesia with $15 \mathrm{ml}$ of $0.75 \%$ Ropivacaine. After completion of surgery and recovery of anesthesia, the patient was shifted to post-operative ward. The patients in whom surgical time exceeded two hours were excluded from the study. On first demand of analgesia(VAS $>3$ ), epidural bolus of $10 \mathrm{ml}$ of $0.2 \%$ Ropivacaine was given in group I and $10 \mathrm{ml}$ of $0.2 \%$ Levobupivacaine in group II followed by continuous infusion of respective drugs @ $6 \mathrm{ml} /$ hour in either groups via elastomeric pump to maintain adequate block. Onset of pain relief was assessed by Visual Analogue Scale (VAS).
Pain was assessed by using 10 point Visual Analogue Scale (VAS) in which a score of " 0 " indicated "no pain" and score of "10" "worst pain imaginable". The VAS measurements were obtained from the end of surgery to the onset of block and then every 2 hours for first 6 hours and then 4 hourly till 24 hours. A Bromage Scale for the lower extremities was used to assess motor function. This scale consists of the following four scores:

i. Free movement of legs and feet.

ii. Just able to flex knees with free movement of feet.

iii. Unable to flex knees but with free movement of feet.

iv. Unable to move legs or feet.

Sensory blockade was assessed using pinprick and cold sensation using alcohol swabs. Motor block duration was the time for return to Bromage Scale I. The highest sensory block level and recovery time of both sensory and motor block was recorded. Adverse effects like nausea, vomiting, shivering was also documented and managed symptomatically. Hypotension (defined by decrease in MAP below $20 \%$ of baseline of SBP $<90 \mathrm{~mm} \mathrm{Hg}$ ) was to be treated by injection Mephentermin intravenous 6mg. Bradycardia (HR $<50 \mathrm{bpm}$ ) was to be treated by atropine IV $0.6 \mathrm{mg}$. Respiratory depression ( $\mathrm{RR}<8$ breaths per min or $\mathrm{SpO} 2<95 \%)$ was to be treated by oxygen supplementation and respiratory support if required. Times for Recording

a. T0: Before starting epidural infusion

b. T1: Onset of block

c. T2: 2 hours after administration of drug

d. T3: 4 hours after administration of drug

e. T4: 6 hours after administration of drug

f. T5: 12 hours after administration of drug

g. T6: 18 hours after administration of drug

h. T7: 24 hours after administration of drug

\section{Statistical Tools Employed}

The statistical analysis was done using SPSS (Statistical Package for Social Sciences) Version 15.0 statistical Analysis Software. The values were represented in Number (\%) and Mean \pm SD. Block characteristics were analyzed using Mann Whitney U test.

\section{Results}

Demographic data was comparable with respect to age, gender distribution and ASA status (Table $1 \& 2$ ). Mean age of Group I $(65.32+4.00$ years) was found to be slightly lower than that of Group II $(65.57+4.26$ years) but this difference was not found to be statistically significant ( $\mathrm{p}=0.822$ ). Out of 56 patients enrolled in the study, majority $(n=38 ; 67.86 \%)$ were ASA Grade II and rest 18 (32.14\%) were ASA grade III. The difference in ASA grade was not found to be statistically significant $(\mathrm{p}=1.000)$. On evaluating the hemodynamic effect of two drugs, no statistically significant difference was observed between two groups(table 3).

With respect to motor block achieved in both groups, at baseline, level was of higher order in Group I as compared to Group II but this difference was not found to be statistically significant $(\mathrm{p}=0.185)$. At all the other time intervals motor blockade of Group II was of higher 
order as compared to Group I and this difference was found to be very highly statistically significant $(\mathrm{p}<0.05)$ for all time intervals except at the time of onset (Table 4)(Figure 1).

On comparing the sensory block between the groups, a higher level was achieved in group I as compared to group IIat all time intervals and difference was found to be statistically significant at all time intervals except at baseline (Table 5)(Figure 2). No complication of nausea, vomiting and hypotension was reported in any of the patient At baseline, before starting the infusion, VAS score 3 was found in higher proportion in Group I (60.71\%) as compared to Group II $(50.0 \%)$, rest of the patients had VAS score 4 at baseline. Difference in VAS score of both the groups was not found to be statistically significant $(\mathrm{p}=0.420)$ (Table 6).

Time taken to achieve VAS score 0 (onset of action) was $5.46 \pm 0.84$ min in Group I as compared to $16.07 \pm 1.78 \mathrm{~min}$ in Group II, thus showing the difference to be very highly significant $(\mathrm{p}<0.001)$ (Table 7). After stopping the infusion, duration of analgesia was significantly higher in Group I as compared to Group II ( $\mathrm{p}=0.004)$ (Table 8).

Table I Demographic Profile of Study Population

\begin{tabular}{lllllll}
\hline Age Group & & & & & \\
\hline $60-70$ & 26 & 92.86 & 26 & 92.86 & 0 & 1 \\
$>70$ & 2 & 7.14 & 2 & 7.14 & & \\
Mean Age $\pm \mathrm{SD}$ (Range) (Range) & $65.32+4.00$ & $(60-75)$ & $65.57+4.26$ & $(60-75)$ & ' $\mathrm{t}$ '=0.226; $\mathrm{p}=0.822$ \\
Gender & & & & & & \\
Female & 8 & 28.57 & 11 & 39.29 & 0.717 & 0.397 \\
Male & 20 & 71.43 & 17 & 60.71 & & \\
\hline
\end{tabular}

Table 2 ASA Grade of Study Population

\begin{tabular}{lllllll}
\hline & \multicolumn{2}{l}{ Group I $(\mathbf{n}=\mathbf{2 8})$} & \multicolumn{2}{l}{ Group II $(\mathbf{n}=\mathbf{2 8})$} & \multicolumn{2}{l}{ Statistical Significance } \\
\cline { 2 - 7 } & No. & $\%$ & No. & $\%$ & c2 & 'p' \\
ASA Grade II & 19 & 67.86 & 19 & 67.86 & 0 & I \\
ASA Grade III & 9 & 32.14 & 9 & 32.14 & 0 & \\
\hline
\end{tabular}

Table 3 Between Group Comparison of Baseline Hemodynamic Variables

\begin{tabular}{lllllll}
\hline & \multicolumn{2}{l}{ Group I $(\mathbf{n = 2 8})$} & \multicolumn{2}{l}{ Group II $(\mathbf{n = 2 8})$} & \multicolumn{2}{l}{ Statistical significance } \\
\cline { 2 - 7 } & Mean & SD & Mean & SD & 't' & 'p' \\
Heart rate (per min) & 79.54 & 3.43 & 80.32 & 5.55 & -0.637 & 0.527 \\
Systolic Blood pressure $(\mathrm{mm} \mathrm{Hg})$ & 129.50 & 4.20 & 130.07 & 4.09 & -0.516 & 0.608 \\
Diastolic Blood Pressure $(\mathrm{mm} \mathrm{Hg})$ & 81.36 & 4.08 & 83.29 & 4.04 & -1.779 & 0.081 \\
\hline
\end{tabular}

Table 4 Between Group Comparison of Motor Blockade. (Bromage score) at different Time intervals (Mann-Whitney Test)

\begin{tabular}{|c|c|c|c|c|c|c|}
\hline \multicolumn{2}{|c|}{ Group I $(n=28)$} & \multicolumn{2}{|c|}{ Group II $(n=28)$} & \multicolumn{2}{|c|}{ Statistical Significance } & \\
\hline Mean & SD & Mean & SD & c2 & 'p' & \\
\hline Baseline & 2.61 & 0.50 & 2.43 & 0.50 & 1.325 & 0.185 \\
\hline Onset & 2.43 & 0.50 & 2.50 & 0.51 & 0.531 & 0.595 \\
\hline 2 hours & 1.75 & 0.44 & 2.64 & 0.49 & -5.273 & $<0.001$ \\
\hline 4 hours & 1.00 & 0.00 & 2.82 & 0.39 & -7.158 & $<0.001$ \\
\hline 8 hours & 1.00 & 0.00 & 2.96 & 0.19 & -3.873 & $<0.001$ \\
\hline 12 hours & 1.00 & 0.00 & 2.71 & 0.46 & -2.324 & 0.020 \\
\hline 18 hours & 1.00 & 0.00 & 2.71 & 0.46 & -2.324 & 0.020 \\
\hline 24 hours & 1.00 & 0.00 & 2.71 & 0.46 & -2.324 & 0.020 \\
\hline
\end{tabular}

Table 5 Between Group Comparison of Level of sensory analgesia at different Time intervals (Mann-Whitney Test)

\begin{tabular}{|c|c|c|c|c|c|c|c|c|}
\hline \multicolumn{3}{|c|}{ Group I $(n=28)$} & \multicolumn{3}{|c|}{ Group II (n=28) } & \multicolumn{3}{|c|}{ Statistical Significance } \\
\hline Median & Mean & SD & Median & Mean & SD & $\mathbf{z}$ & 'p' & \\
\hline Baseline & 0 & 0 & 0.00 & 0 & 0 & 0.00 & 0.000 & 1.000 \\
\hline Onset & 8 & 7.43 & 1.07 & 8 & 8.79 & 0.99 & -4.118 & $<0.001$ \\
\hline 2 hours & 8 & 7.93 & 0.66 & 10 & 9.00 & 2.00 & -4.456 & $<0.001$ \\
\hline 4 hours & 8 & 8.18 & 0.94 & 10 & 9.64 & 0.78 & -4.956 & $<0.001$ \\
\hline 8 hours & 9 & 8.96 & 1.00 & 10 & 10.00 & 0.54 & -4.127 & $<0.001$ \\
\hline 12 hours & 10 & 9.46 & 0.88 & 10 & 10.93 & 1.02 & -4.605 & $<0.001$ \\
\hline 18 hours & 10 & 10.07 & 0.38 & 12 & 11.64 & 0.78 & -5.887 & $<0.001$ \\
\hline 24 hours & 12 & 11.50 & 0.88 & 12 & 12.00 & 0.00 & -2.803 & 0.005 \\
\hline
\end{tabular}


Table 6 VAS Score at Baseline in Study Population

\begin{tabular}{|c|c|c|c|c|c|}
\hline \multirow{2}{*}{ AS Score } & \multicolumn{2}{|c|}{ Group I $(n=28)$} & Group II $(n=28)$ & \multicolumn{2}{|c|}{ Statistical Significance } \\
\hline & No. & $\%$ & No. & $\%$ 't' & 'p' \\
\hline 3 & 17 & 60.71 & 14 & 50 & \\
\hline 4 & 11 & 39.29 & 14 & $50^{0.65}$ & 0.42 \\
\hline
\end{tabular}

Table 7 Time taken to achieve VAS score $0(\mathrm{~min})$

\begin{tabular}{llllll}
\hline \multicolumn{2}{l}{ Group I (n=28) } & \multicolumn{2}{l}{ Group II (n=28) } & \multicolumn{2}{l}{ Statistical significance } \\
Mean & SD & Mean & SD & 't' & ' $\mathbf{~ '}$ \\
5.46 & 0.84 & 16.07 & 1.78 & 28.486 & $<0.00$ I \\
\hline
\end{tabular}

Table 8 Duration of analgesia after stopping the infusion (hour)

\begin{tabular}{llllll}
\hline Group I $(\mathbf{n}=\mathbf{2 8})$ & & Group II $(\mathbf{n}=\mathbf{2 8})$ & \multicolumn{2}{c}{ Statistical significance } \\
\hline Mean & SD & Mean & SD & 't' & 'p' \\
2.28 & I.84 & 1.07 & 0.89 & 3.133 & 0.004
\end{tabular}

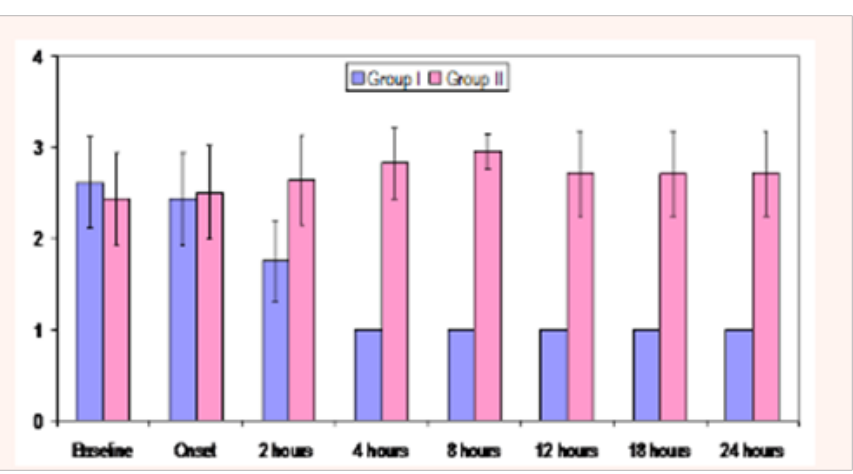

Figure I Comparison of Motor Blockade.

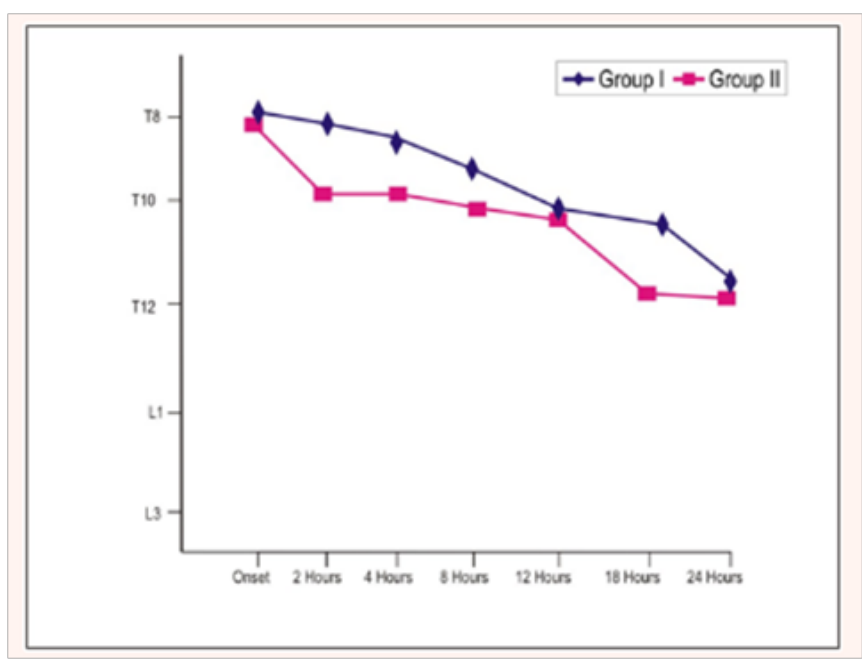

Figure 2 Comparison of Level of sensory analgesia.

\section{Discussion}

Perception of pain is sometimes enigmatic and different among elderly population owing to presence of comorbid conditions and impaired physiological and cognitive status and a paradox of endurance and irritation. Hence, post-operative pain management among elderly patients is a challenging and daunting task for anesthesiologists. It is desirous that while pain is resolved even when it is expressed silently at the same time the management of pain should not intermingle with any comorbid condition of the patient and give rise to side effects. In effect, the post-operative pain management in elderly patients is just like walking on the tight rope and an anesthetist is expected to take all the precautions while walking from one end of the rope to the other.

Traditionally used analgesics like opioids have a known risk of adverse effects in geriatric patients. Moreover, in elderly patients with cardiopulmonary and other systemic co-morbidities it is sometimes not possible to follow the routine protocol for pain management owing to their interference with metabolic functions. In the recent years, regional anesthesia has emerged as one of the preferred and convenient mode of post-operative analgesic management. In elderly patients with systemic co-morbidities, continuous epidural analgesia effectively manages postoperative pain, allows early ambulation and reduces mortality by decreasing deep vein thrombosis and thromboembolism ${ }^{3}$

Among traditionally used regional anesthetics, bupivacaine is one of the most popular but owing to its reported cardio toxicity, it happens to be less preferred among elderly patients with increased cardiovascular risk. As a substitute, two less cardiotoxic isomers of bupivacaine - Ropivacaine and Levobupivacaine have emerged as a viable option. . The levorotatory isomers were shown to have a safer pharmacological profile, ${ }^{4,5}$ with less cardiac and neurotoxic adverse effects, ${ }^{6}$ Both the drugs have shown a comparable analgesic activity in different studies when used through variable routes using variable dosages.$^{7-9}$ However, there are limited and almost no studies available among elderly population despite an evidence indicating that pharmacology of these drugs is affected by age. ${ }^{10}$

With this background, the present study was carried out with an aim to evaluate and compare the efficacy of ropivacaine and levobupivacaine as a continuous epidural post-operative analgesia in elderly patients with cardiopulmonary co-morbidities. For this purpose, a total of 56 elderly patients with cardiopulmonary comorbidities of ASA grade II or grade III, scheduled to undergo elective lower limb orthopaedic surgeries falling in the sampling frame were enrolled in the study.Out of these 56 patients, a total of $28(50 \%)$ patients were managed using Ropivacaine (Group I) while remaining $28(50 \%)$ were given infusion of Levobupivacaine (Group II). On evaluating the hemodynamic effect of two drugs, no statistically significant difference was observed between two groups (Table 8).

Thus overall, both the groups indicated a safe hemodynamic profile and were in accordance with their experimentally and clinically described characteristic of being free of cardiotoxic effect. ${ }^{11}$ In present study, no vasopressor was used in any case in either of two 
groups. With respect to motor block, motor block (Bromage scale) achieved, in both the groups, was above 2 at baseline and maintained at onset also. In Levobupivacaine group, a continuous increase in intensity of motor block was observed till eight hours, which receded between Bromage II to III till twenty-four hours. Whereas in Ropivacaine group, the motor block level receded to score 1 at 4 hours and thereafter remained stable till the end of study period. This was a situation where Ropivacaine definitely had an upper edge as it ensured early motor recovery. Ropivacaine produces effects similar to other local anaesthetics via reversible inhibition of sodium ion influx in nerve fibres. Ropivacaine is less lipophilic than bupivacaine and is less likely to penetrate large myelinated motor fibres, resulting in a relatively reduced motor blockade. Thus, ropivacaine has a greater degree of motor sensory differentiation, which could be useful when motor blockade is undesirable. ${ }^{12,13}$ Definitely on this point ropivacaine scored over levobupivacaine. Senardet al. ${ }^{8}$ showed achievement of Bromage score of 1 for all patients after the fourth postoperative hour and observed more patients in Ropivacaine group were able to ambulate as compared to levobupivacaine group with no concerning side effect in any study group. In our study also the group I (Ropivacaine), all patients achieved Bromage I after fourth post-operative hour and motor block was intense in levobupivacaine group as compared to Ropivacaine group which is in accordance with the above study.

In present study, ropivacaine achieved higher level of sensory block at all time intervals starting from onset itself. This could be again attributed to the selective motor-sensory differentiation of ropivacaine. The achievement of higher level of sensory block in ropivacaine group can be attributed to the continuous infusion and faster action of ropivacaine to achieve T8 dermatome level. Considering the fact that the epidural absorption of levobupivacaine gets affected by age as the fraction absorbed decreases and the fast absorption phase is shorter in older (aged $>70$ years) compared with the younger (aged 18-44 years) patients and this could be the reason both for slower action and lower dermatome level achieved by leveobupivacaine. ${ }^{14}$

In present study, at baseline VAS scores in two groups were matched. However, time taken to achieve score 0 was much lower in ropivacaine group as compared to levobupivcaine group while duration of analgesia was longer in ropivacaine group as compared to levobupivacaine group. The slower achievement of VAS score 0 in levobupivacaine group could be attributed to the slower initial absorption process for levobupivacaine in elderly patients. ${ }^{10}$ Almost all the studies in literature have shown comparable analgesic effect of ropivacaine and Levobupivacaine ${ }^{15}$ although in present study, as none of the patients in either groups required rescue analgesic throughout the infusion period, we can assume that there existed no difference between two groups with respect to level of pain control. However, the prolongation of analgesic effect in ropivacaine group as compared to levobupivacaine group could mainly be attributed to higher level of dermatome achieved and time taken to recede to lower sensory block levels where the perception of pain became strong enough to call for rescue analgesic.

The findings in present study showed a comparable profile of ropivacaine and levobupivacaine on most of the counts from clinical point of view, both provided excellent analgesic effect, a good hemodynamic stability and complication free infusion. However, from the critical point of view taking into account the statistical differences, levobupivacaine seemed to be poor as compared to ropivacaine. As compared to levobupivacaine, ropivacaine had an early motor block recovery, early achievement of VAS score 0 , ability to achieve higher levels of block and finally prolongation of analgesic effect even after the infusion was stopped. The findings in present study are specific in view of the specific population they addressed, there is no doubt that cognition issues related with objective scoring of VAS, loss of tactile sensation owing to probable systemic illnesses and growing age might have a confounding effect on the results.

\section{Conclusion}

In elderly patients with systemic co-morbidities, continuous epidural analgesia is an effective measure in managing postoperative pain. Regional anesthesia (spinal/epidural) reducesmortality in orthopedic surgeries by decreasing deep vein thrombosis and thromboembolism. From our study we can conclude that both levobupivacine $(0.2 \%)$ and ropivacaine $(0.2 \%)$ can be used as continuous epidural infusion owing topost-operative pain free period with stable hemodynamic profile and no side effect. It can be concluded that ropivacaine seemed to be a better option and is preferred over levobupivacaine for continuous epidural infusion in elderly patients with cardio pulmonary abnormalities on account of early motor recovery and longer duration of analgesia after stopping infusion. The findings in present study are specific in view of the specific population they addressed, there is no doubt that cognition issues related with objective scoring of VAS, loss of tactile sensation owing to probable systemic illnesses and growing age might have a confounding effect on the results. We would like to recommend more studies in a larger sample size and probably under varied clinical situations to substantiate these findings with substantial clinical evidence.

\section{Conflicts of Interest}

The authors do not have any Conflict of interests.

\section{Acknowledgments}

None.

\section{Funding}

None.

\section{References}

1. Kinsella K, He W. An Ageing World: 2008. Census Bureau, Washington, DC, USA. 2009

2. Ornstein E, Matteo RS. Effects of opioids. In: Mcleskey CH (Ed): Geriatric Anesthesiology. Baltimore, Williams \& Wilkins; USA, 1997; pp. 249-260.

3. Sharrock NE, Haas SB, Hargett MJ, et al. Effects of epidural Anaesthesia on the incidence of deep vein thrombosis after total knee replacement. $J$ Bone Joint Surg. 1991;73(4):502-506.

4. McLeod GA, Burke D. Levobupivacaine. Anaesthesia. 2001;56:331-41.

5. Casati A, Baciarello M. Enantiomeric local anesthetics: Can ropivacaine and levobupivacaine improve our practice? Curr Drug Ther. 2006;1(1):85-89.

6. Huang YF, Pryor ME, Mather LE, et al. Cardiovascular and central nervous system effects of intravenous levobupivacaine and bupivacaine in sheep. Anesth Analg. 1998;86(4):797-804.

7. De Negri P, Ivani G, Tirri T, et al. A comparison of epidural bupivacaine, levobupivacaine, and ropivacaine on postoperative analgesia and motor blockade. Anesth Analg. 2004;99(1):45-48.

8. Senard M, Kaba A, Jacquemin MJ, et al. Epidural Levobupivacaine $0.1 \%$ or Ropivacaine $0.1 \%$ Combined with Morphine Provides Comparable Analgesia After Abdominal Surgery. Anesth Analg. 2004;98(2):389394 
9. Kountoudi M, Papadaki E, Pandazi A, et al. A comparison of epidural levobupivacaine $0.5 \%$ with ropivacaine $0.5 \%$ for inguinal hernia repair procedure: blood pressure and heart rate alterations European Journal of Anaesthesiology. 2004;21:103.

10. Simon, Mischa JG. Epidural anaesthesia with levobupivacaine and ropivacaine: effects of age on the pharmacokinetics, neural blockade and haemodynamics (Doctoral thesis). Department Anesthesiology, Faculty of Medicine / Leiden University Medical Center (LUMC), Leiden University, Netherlands. 2006

11. Burlacu CL, Buggy DJ. Update on local anesthetics: focus on levobupivacaine. Ther Clin Risk Manag. 2008;4(2):381-392.
12. Hansen TG. Ropivacaine: a pharmacological review. Expert Rev Neurother. 2004;4(5):781-791.

13. Kuthiala G, Chaudhary G. Ropivacaine: A review of its pharmacology and clinical use. Indian J Anaesth. 2011;55(2): 104-10.

14. Bajwa SS, Kaur J. Clinical profile of levobupivacaine in regional anesthesia: A systematic review. J Anaesthesiol Clin Pharmacol. 2013;29(4):530-539.

15. Bicer C, Eskitascioglu T, Aksu R, et al. Comparison of Preincisional Infiltrated Levobupivacaine and Ropivacaine for Acute Postoperative Pain Relief After Septorhinoplasty. Curr Ther Res Clin Exp. 2011;72(1):13-22. 